\title{
Catecholamines Are Required for the Acquisition of Secretory Responsiveness by Sweat Glands
}

\author{
Hua Tian, ${ }^{1}$ Beth Habecker, ${ }^{1}$ Guy Guidry, ${ }^{1}$ Allan Gurtan, ${ }^{1}$ Maribel Rios, ${ }^{2}$ Suzanne Roffler-Tarlov, ${ }^{2}$ and \\ Story C. Landis ${ }^{1}$
}

${ }^{1}$ National Institute of Neurological Disorders and Stroke, National Institutes of Health, Bethesda, Maryland 20892, and

2Departments of Neuroscience and Anatomy and Cell Biology, Tufts University Medical School, Boston,

Massachusetts 02111

The sympathetic innervation of sweat glands undergoes a developmental change in transmitter phenotype from catecholaminergic to cholinergic. Acetylcholine elicits sweating and is necessary for development and maintenance of secretory responsiveness, the ability of glands to produce sweat after nerve stimulation or agonist administration. To determine whether catecholamines play a role in the development or function of this system, we examined the onset of secretory responsiveness in two transgenic mouse lines, one albino and the other pigmented, that lack tyrosine hydroxylase (TH), the rate-limiting enzyme in catecholamine synthesis. Although both lines lack $\mathrm{TH}$, their catecholamine levels differ because tyrosinase in pigmented mice serves as an alternative source for catecholamine synthesis (Rios et al., 1999). At postnatal day 21 (P21), 28 glands on average are active in interdigital hind footpads of albino $\mathrm{TH}$ wild-type mice. In contrast, fewer than one gland is active in albino $\mathrm{TH}$ null mice, which lack catecholamines in gland innervation. Treatment of albino TH null mice with DOPA, a catecholamine precursor, from P11 to P21 increases the number of active glands to 14. Pigmented $\mathrm{TH}$ null mice, which have faint catecholamine fluorescence in the developing gland innervation, possess 12 active glands at P21, indicating that catecholamines made via tyrosinase, albeit reduced from wild-type levels, support development of responsiveness. Gland formation and the appearance of cholinergic markers occur normally in albino TH null mice, suggesting that catecholamines act directly on gland cells to trigger their final differentiation and to induce responsiveness. Thus, catecholamines, like acetylcholine, are essential for the development of secretory responsiveness.

Key words: synapse development; transmitter plasticity; sweat glands; sympathetic neuron; acetylcholine; catecholamines; tyrosinase; tyrosine hydroxylase null
The sympathetic innervation of sweat glands is unusual in that the postganglionic fibers release acetylcholine rather than norepinephrine, the transmitter used at other sympathetic synapses. The gland innervation contains choline acetyltransferase (ChAT), the synthetic enzyme for acetylcholine, and the vesicular acetylcholine transporter (VAChT) but not catecholamines (Landis and Keefe, 1983; Leblanc and Landis, 1986; Weihe et al., 1996). Muscarinic agonists mimic the ability of nerve stimulation to elicit sweating, and nerve-evoked sweating is blocked by muscarinic antagonists (Langley, 1922; Hayashi and Nakagawa, 1963; Stevens and Landis, 1987; Vilches et al., 1995).

In addition to eliciting sweat secretion in adult rodents, cholinergic transmission is required for the development and maintenance of secretory responsiveness, the ability of glands to produce sweat after nerve stimulation or agonist administration. During development, secretory responsiveness develops after cholinergic properties appear in the innervation and fails to develop when innervation is absent (Stevens and Landis, 1988). In adults, denervation causes loss of responsiveness, and reinnervation restores it (Hayashi and Nakagawa, 1963; Kennedy and Sakuta, 1984; Grant et al., 1991). The effects of innervation on secretory responsiveness are mediated by acetylcholine (Grant et al., 1995). Acquisition of responsiveness in young animals is blocked by a 1-week-long treatment with muscarinic antagonists; secretory responsiveness disappears in adults treated for 1 week with muscarinic antagonists,

Received June 1, 2000; accepted June 28, 2000.

These studies were supported by National Institutes of Health Grant NS35639 and the intramural research program at the National Institute of Neurological Disorders and Stroke. We thank Dr. Dona Chikaraishi for providing mating pairs of the TH and tyrosinase-deficient mice, Dr. Ling Hou for help with the tyrosinase assay, and Drs. Nicole Francis and Graeme Eisenhofer for thoughtful suggestions

Correspondence should be addressed to Story C. Landis, National Institute of Neurological Disorders and Stroke, National Institutes of Health, Bethesda, MD 20892. E-mail: landiss@ninds.nih.gov.

Copyright (C) 2000 Society for Neuroscience $0270-6474 / 00 / 207362-08 \$ 15.00 / 0$ and responsiveness is maintained by muscarinic agonists after denervation.

During development, however, the sympathetic innervation of sweat glands is catecholaminergic and not cholinergic (Landis and Keefe, 1983; Leblanc and Landis, 1986; Landis et al., 1988; Guidry and Landis, 1998). The initial innervation contains catecholamines and immunoreactivity for the catecholamine synthetic enzymes tyrosine hydroxylase $(\mathrm{TH})$ and dopamine $\beta$-hydroxylase $(\mathrm{D} \beta \mathrm{H})$. During the second and third postnatal weeks, catecholaminergic properties decrease, and cholinergic and peptidergic properties appear. This change is retrogradely specified by a cholinergic differentiation factor produced by sweat glands. In tabby mutant mice, which lack sweat glands, sympathetic axons grow to the region where glands normally form, retain catecholamines, and do not acquire cholinergic properties (Guidry and Landis, 1995). Conversely, when sweat gland primordia are transplanted in place of hairy skin, sympathetic axons innervating the transplanted glands lose catecholamines and adopt a cholinergic phenotype (Schotzinger and Landis, 1988).

Because catecholamines do not elicit sweating in developing or adult rodents (Stevens and Landis, 1987), it has been unclear whether the initial catecholaminergic innervation is functional. It is possible that catecholamines play a developmental role in sweat gland differentiation or synapse formation. Consistent with this possibility, in culture catecholamines are required to induce sweat glands to produce cholinergic differentiation factor (Habecker and Landis, 1994; Habecker et al., 1995). To determine whether catecholamines play a role in the development of synapses between sympathetic neurons and sweat glands in vivo as they do in vitro, Tsahai Tafari et al. (1997) examined sweating in mutant mice that lack either $\mathrm{TH}$, which converts tyrosine to DOPA, or $\mathrm{D} \beta \mathrm{H}$, which converts dopamine to norepinephrine. Although the onset of secretory responsiveness was delayed in these animals, it was not prevented, and Tsahai Tafari et al. (1997) concluded that norepi- 
nephrine is not required for responsiveness and therefore for production of the sweat gland cholinergic factor.

Here we report the results of an examination of secretory responsiveness in mice that lack $\mathrm{TH}$ and are either pigmented or albino (tyrosinase-deficient). This inquiry was prompted by our discovery that tyrosinase in $\mathrm{TH}$ null mice serves as an alternative pathway for catecholamine production, because like $\mathrm{TH}$ it can convert tyrosine to DOPA (Rios et al., 1999). The peripheral tissues of pigmented TH null mice contain between 10 and $22 \%$ of wild-type catecholamines. In contrast, tyrosinase-deficient (albino) $\mathrm{TH}$ null mice produce $<0.3 \%$ of wild-type catecholamines. We find that secretory responsiveness does not develop in the albino $\mathrm{TH}$ null mice, indicating that catecholamines, like acetylcholine, are essential for the acquisition of secretory responsiveness. Surprisingly, catecholamines are required not for the appearance of cholinergic properties in the gland innervation but rather to trigger functional maturation of the glands.

\section{MATERIALS AND METHODS}

Animals. Heterozygous breeding pairs of pigmented or albino $\mathrm{TH}$ null mice were crossed to produce $\mathrm{TH}$ null, heterozygous, and wild-type animals that were either pigmented or albino. The pigmented mice heterozygous for the $\mathrm{TH}$ mutation were created by homologous recombination in embryonic stem cells (Rios et al., 1999). The albino mice heterozygous for the TH mutation and lacking tyrosinase were obtained by back-crossing the $\mathrm{TH}+/-$ mice on the original pigmented background onto an albino [homozygous tyrosinase (c-locus)-deficient] ICR background for three generations as described (Rios et al., 1999). Because catecholamines are required for normal fetal development (Kobayashi et al., 1995; Zhou et al., 1995; Rios et al., 1999), breeding pairs, both pigmented and albino, were provided with L-DOPA (Sigma, St. Louis, MO) in their drinking water at a final concentration of $1.0 \mathrm{mg} / \mathrm{ml}$. Water containing the drug and $0.25 \%$ ascorbic acid, to reduce oxidation, was shielded from light and changed daily. Administration of the catecholamine precursor was discontinued at the time of birth. TH null pups survive without further supplementation for up to 3 weeks. TH genotypes were determined from PCR analysis of DNA isolated from tails.

To determine whether supplementation with L-DOPA during the second and third postnatal weeks could rescue the secretory response as it does for movement and feeding in dopamine-deficient mice (Zhou and Palmiter, 1995), we treated four albino TH null pups with L-DOPA from postnatal day 11 (P11) to P21. The pups were injected intraperitoneally twice daily with $75 \mathrm{mg} / \mathrm{kg}$ L-DOPA dissolved in saline solution containing $0.25 \%$ ascorbic acid.

To examine the effects of tyrosinase on the acquisition of the sweating response, we compared two types of mice that were genetically identical except at the tyrosinase locus. The comparison was made between pigmented C57BL/6J and albino C57BL/6J mice (C57BL/6J-Tyr ${ }^{\mathrm{c}-2 \mathrm{~J}}$; Jackson Laboratories, Bar Harbor, ME). The albino C57BL/6J mice, like ICR mice, carry a mutation in the tyrosinase gene that eliminates the activity of the enzyme. Heterozygous breeding pairs were maintained, and albino $(-/-)$ or pigmented $(+/-$ or $+/+)$ littermates were examined for secretory responsiveness from $\mathrm{P} 15$ to $\mathrm{P} 60$.

Sweating assay. To assay secretory responsiveness, anesthetized mice were treated with a muscarinic cholinergic agonist, and sweating was visualized with a dental impression method (Kennedy et al., 1984). Mice were anesthetized with Avertin $(0.02 \mathrm{ml} / \mathrm{gm}$ body weight, tribromoethanol and tert-amyl alcohol; Sigma) and then treated with pilocarpine $(3.0 \mathrm{mg} / \mathrm{kg}$, i.p.; Sigma). Dental impression material (Elasticon; Kerr Co., Romulus, MI) was subsequently applied to both hind feet and allowed to polymerize. Two molds were made from each hind foot, and active pores were counted in the two interdigital footpads. Five mice of each genotype were assayed at each age examined. The number of pores was averaged for each mouse, and then the values for the five mice were averaged.

Histochemistry and immunocytochemistry. Catecholamine-containing fibers were identified using the glyoxylic acid method (de La Torre, 1980). Ten micrometer cryostat sections of fresh frozen footpads were melted onto slides and dipped in a solution containing 1\% glyoxylic acid (Sigma), $0.2 \mathrm{M}$ potassium phosphate, and $0.2 \mathrm{M}$ sucrose, $\mathrm{pH}$ 7.4. The sections were dried in an air stream, heated to $95^{\circ} \mathrm{C}$ under mineral oil for $2.5 \mathrm{~min}$, and coverslipped. Acetylcholinesterase histochemistry was performed on perfusion-fixed tissue sections as described previously (Landis and Keefe, 1983).

Tyrosinase enzyme activity was detected using the DOPA reaction (Gurr, 1958). Sections of footpads and lumbar ganglia from paraformaldehyde-fixed mice were rinsed with PBS, incubated in $0.2 \%$ DOPA in PBS overnight at $37^{\circ} \mathrm{C}$, and then coverslipped with $50 \%$ glycerol in PBS. In some cases, the sections were counterstained with methylene blue.

$\mathrm{Na}^{+}-\mathrm{K}^{+}$ATPase activity was demonstrated using the histochemical assay developed by Guth and Albers (1974) with a few modifications. For this study, footpads were frozen without fixation, and sections were mounted on gelatinized slides. We used the ammonium salt of $p$-nitrophenyl phosphate (Sigma) and inhibited enzyme activity with a final concentration of $3 \mu \mathrm{M}$ ouabain (Sigma).

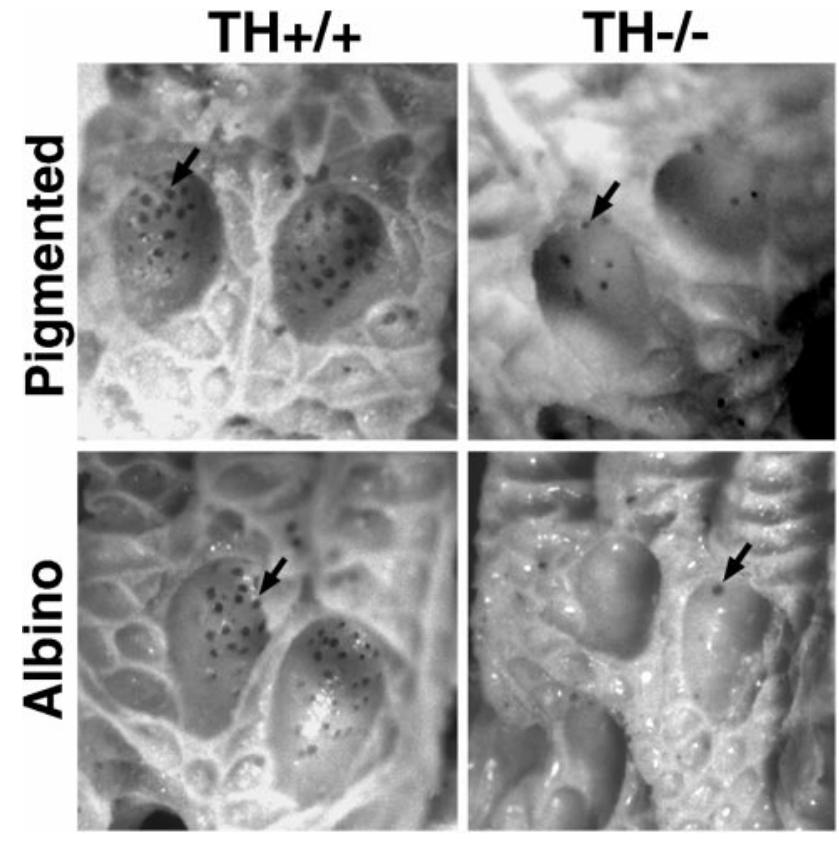

Figure 1. Representative molds from sweating assays. Sweating was induced in anesthetized P21 mice by treatment with the muscarinic agonist pilocarpine, and molds were made of the hind footpads with a dental impression material. Each active gland appears as a dark dot (arrows). Many glands are active in the interdigital pads of both pigmented and albino TH wild-type mice. Approximately one-third of the normal number of glands can be detected in the pads of pigmented TH null mice. Only a rare gland is active in the pads of the albino $\mathrm{TH}$ null mice.

For immunolabeling, deeply anesthetized mice were perfused with $4 \%$ paraformaldehyde for $10 \mathrm{~min}$. Footpads were dissected and immersed in fixative for 1 additional hour at room temperature, rinsed in PBS, and then equilibrated with $30 \%$ sucrose in $0.1 \mathrm{~m}$ phosphate buffer at $4^{\circ} \mathrm{C}$. Ten to twelve micrometer cryostat sections were incubated for $1 \mathrm{hr}$ at room temperature in dilution buffer containing $2 \%$ bovine serum albumin, $0.3 \%$ Triton X-100, and $0.1 \%$ sodium azide in PBS. Sections were incubated overnight with primary antisera in dilution buffer, rinsed in PBS, and labeled with species-specific secondary antisera in dilution buffer containing 5\% rat serum for $2 \mathrm{hr}$. Secondary antibodies were conjugated to Oregon Green 514 (Molecular Probes, Eugene, OR) or lissaminerhodamine (Jackson ImmunoResearch, West Grove, PA). Rabbit antiVAChT was generously provided by A. Roghani (Texas Tech University Health Sciences Center, Lubbock, TX) (Roghani et al., 1998). Guinea pig anti-vasoactive intestinal peptide (VIP) was generated in our laboratory (Tyrrell and Landis, 1994), and rabbit anti-calcitonin gene-related peptide (CGRP) was obtained from Amersham Pharmacia Biotech (Arlington Heights, IL). The aquaporin 5 polyclonal antiserum as provided by Dr. Bruce Baum (National Institute of Dental and Craniofacial Research, National Institutes of Health, Bethesda, MD) (He et al., 1997). To localize smooth muscle actin, $5 \mu \mathrm{m}$ cryostat sections were incubated with mouse anti-smooth muscle actin directly conjugated to Cy3 (Sigma) in dilution buffer for $2 \mathrm{hr}$ at room temperature. The labeled sections were rinsed and coverslipped with $50 \%$ glycerol in PBS.

\section{RESULTS}

\section{Catecholamines are required for the development of secretory responsiveness by sweat glands}

To determine whether the acquisition of secretory responsiveness by sweat glands depends on the presence of catecholamines in the early innervation, we examined the development of sweating in albino (tyrosinase-deficient) $\mathrm{TH}$ wild-type and albino $\mathrm{TH}$ null mice. Sweating was elicited in anesthetized mice by systemic injection of pilocarpine, a cholinergic agonist, and assayed by making molds of the hind feet with a dental impression material (Fig. 1). The sweating response was measured by counting pores formed in the molds by sweat droplets produced from active glands (Fig. 2). In albino $\mathrm{TH}$ wild-type mice, at P15, the first age examined, seven glands were active on average in the two interdigital footpads of each hind foot. By P21, 28 glands were active. In marked contrast to the development of the secretory response in albino TH wild-type mice, only a rare active gland was detected in the interdigital foot- 


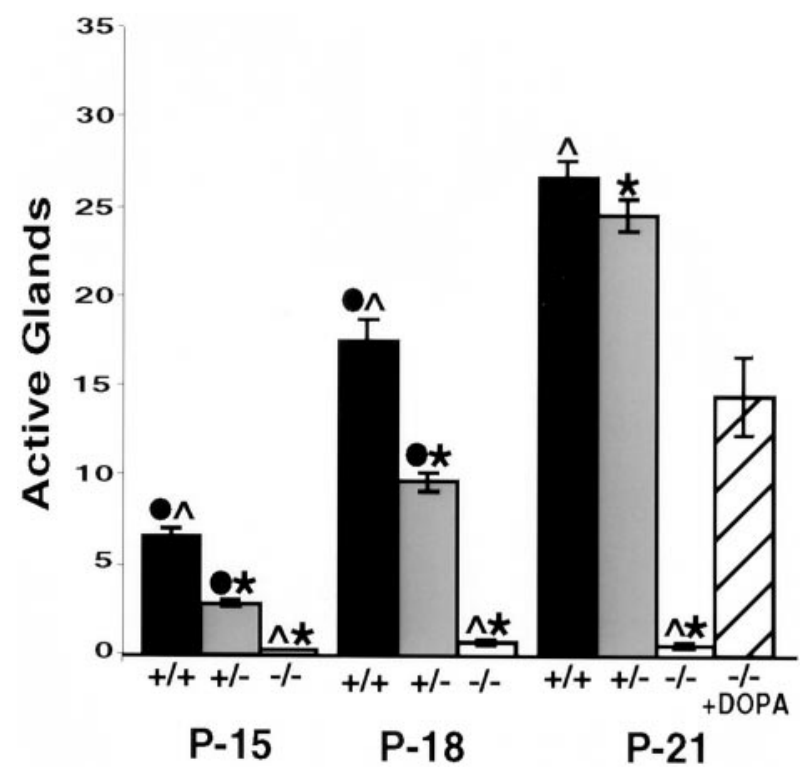

Figure 2. Quantitation of secretory responsiveness in albino TH null mice. The number of active glands was determined by counting the pores formed in the two interdigital footpads of albino mice that were wild-type at the TH locus $(+/+)$, heterozygous for the TH mutation $(+/-)$ or TH null $(-/-)$ at the ages indicated. At P15 and P18, fewer glands were active in the $\mathrm{TH}+/-$ mice than in wild-type mice. At all ages, fewer than one active gland on average was detected in the TH null mice. Four albino TH null pups were injected twice daily with $75 \mathrm{mg} / \mathrm{kg}$ L-DOPA dissolved in saline solution containing $0.25 \%$ ascorbic acid as an antioxidant. When secretory responsiveness was assayed in the DOPA-treated albino TH null mice at P21, 15 glands on average were active. Error bars represent SEM. ANOVA and post hoc pairwise comparisons with Sheffe's $F$ test $(\alpha=0.05$ for all tests) indicated significant differences among all three genotypes at P15 and P18 and between TH null and TH wild-type, heterozygote mice at P21. Secretory responsiveness in the DOPA-treated $\mathrm{TH}$ null mice was significantly different from that in all three untreated groups at P21 (data not shown). Significant differences are indicated as 0 , between $+/+$ and $+/-; \wedge$, between $+/+$ and $-/-$; $*$, between $+/-$ and $-/-$.

pads of albino TH null mice at P15 (Fig. 2). Furthermore, the number of active glands did not increase with age as in wild-type mice.

The absence of catecholamines from the developing sweat gland innervation of albino $\mathrm{TH}$ null mice was confirmed by glyoxylic acid-induced catecholamine histofluorescence. We examined the hind footpads of P7 and P10 wild-type and $\mathrm{TH}$ null mice for catecholamine-containing fibers (Fig. 3). As expected, brightly fluorescent fibers were observed in association with the sweat glands of albino TH wild-type mice at both ages. In contrast, catecholamine histofluorescence was not detectable in the sweat gland innervation of albino $\mathrm{TH}$ null mice at either age.

To determine whether treatment of albino $\mathrm{TH}$ null mice with the catecholamine precursor DOPA would rescue the development of secretory responsiveness, we injected four pups with DOPA from P11 to P21 and then assayed secretory responsiveness on P21. We found that this treatment resulted in the acquisition of secretory responsiveness by approximately half the normal number of glands (Fig. 2).

\section{The sweating response in pigmented $\mathrm{TH}$ null mice is likely attributable to catecholamines present in the developing sweat gland innervation}

When sweating was assayed in pigmented $\mathrm{TH}$ wild-type mice on $\mathrm{P} 15$, we found that 16 glands on average were active in the two interdigital footpads of each hind foot. The number of active glands increased with time in wild-type mice; at P21, an average of 31 glands produced sweat droplets (Figs. 1, 4). It is notable that the secretory response developed more quickly in pigmented $\mathrm{TH}$ wildtype mice than in albino mice (Figs. 2, 4).

In contrast to the pigmented $\mathrm{TH}$ wild-type mice, the pigmented $\mathrm{TH}$ null mice possessed on average one or two active glands in the two interdigital pads on P15. More glands became active with age; 11 pores were detectable at P18, and 12 were detectable at P21
(Figs. 1, 4). Thus, as previously reported by Tsahai Tafari et al. (1997), the appearance of secretory responsiveness is delayed in pigmented $\mathrm{TH}$ null mice, and the sweating response remains reduced compared with that in wild-type mice.

The difference in secretory responsiveness between the two types of TH null pups was striking. Whereas the pigmented $\mathrm{TH}$ null pups acquired secretory responsiveness, albeit delayed in time and reduced in amount when compared with wild-type mice, the albino (tyrosinase-deficient) TH null mice did not (Figs. 2, 4). One explanation for the difference between the two types of $\mathrm{TH}$ null mice is that the sympathetic innervation of sweat glands in the pigmented $\mathrm{TH}$ null mice, like that of the heart and hairy skin (Rios et al., 1999), contains catecholamines. Figure 5 shows the presence of glyoxylic acid-induced catecholamine histofluorescence in the innervation of sweat glands of a pigmented $\mathrm{TH}$ null mouse at P10. Although the catecholamine fluorescence is significantly reduced compared with that in wild-type mice, it is clearly detectable.

Tyrosinase, which like TH converts tyrosine to DOPA, accounts for the presence of catecholamines in pigmented $\mathrm{TH}$ null mice (Rios et al., 1999). Tyrosinase is expressed at high levels in melanocytes. To determine where tyrosinase is localized in footpad skin and to identify melanocytes, we took advantage of the fact that in addition to hydroxylating tyrosine, tyrosinase catalyzes the oxidation of DOPA to dopaquinone, which forms an insoluble black reaction product (Gurr, 1958; Jackson et al., 1994; Spritz and Hearing, 1994; del Marmol and Beermann, 1996). A tyrosinase reaction product was present in melanocytes localized at the intersection of the epidermis and dermis in footpads of pigmented mice (Fig. 6) but not in footpads of albino mice (data not shown). No reaction product could be detected in the sweat gland innervation or in sympathetic neurons in lumbar sympathetic ganglia, which provide innervation to hind footpads in pigmented mice (Fig. 6).

\section{Tyrosinase affects the developmental schedule of the secretory response}

We observed two differences in the appearance of secretory responsiveness in albino and pigmented mice with the same $\mathrm{TH}$ genotype (compare results for wild-type mice in Figs. 2, 4) that raised the possibility that DOPA produced by tyrosinase influences the timing of acquisition of secretory responsiveness in mice that possess active TH. First, approximately half as many glands were active at $\mathrm{P} 15$ in albino $\mathrm{TH}$ wild-type mice as in pigmented $\mathrm{TH}$ wild-type mice. Second, although there was no difference in the secretory responsiveness of pigmented mice that were heterozygous for the $\mathrm{TH}$ null allele $(\mathrm{TH}+/-)$ compared with wild-type mice at P15 or P21, significantly fewer glands were active at all ages assayed in albino mice that were heterozygous for the $\mathrm{TH}$ null allele $(\mathrm{TH}+/-; 3$ at $\mathrm{P} 15,10$ at $\mathrm{P} 18$, and 25 at $\mathrm{P} 21)$.

The finding that tyrosinase influences the development of sweating in wild-type mice was unexpected. It was possible, however, that differences in the genetic backgrounds of the pigmented and albino transgenic mice (Rios et al., 1999), rather than the presence or absence of tyrosinase, affected the development of the sweating response. To examine this possibility, we measured secretory responsiveness in congenic albino and pigmented $\mathrm{C} 57 \mathrm{BL} / 6 \mathrm{~J}$ mice (Fig. 7). Like albino ICR mice, albino C57BL/6J mice carry a mutation in the tyrosinase gene that eliminates the activity of the enzyme. Responsiveness in the albino and pigmented C57BL/6J mice was indistinguishable at P15. At P18 and P21, however, significantly fewer glands were active in albino than in pigmented mice. By P30, this difference had disappeared. Thus, tyrosinase can contribute to the development of secretory responsiveness.

\section{Catecholamines are not required for gland formation}

The secretory tubule of rodent sweat glands forms a coil in the subcutaneous tissue of the footpad and contains two cell types, myoepithelial and secretory cells (Landis and Keefe, 1983; Quick et al., 1984). One possible explanation for the absence of a secretory response in albino TH null mice is that glands fail to form. Examination of footpad sections did not reveal any obvious difference in the appearance of the secretory coils in albino $\mathrm{TH}$ null and albino 


\section{$\mathrm{TH}+/+$}
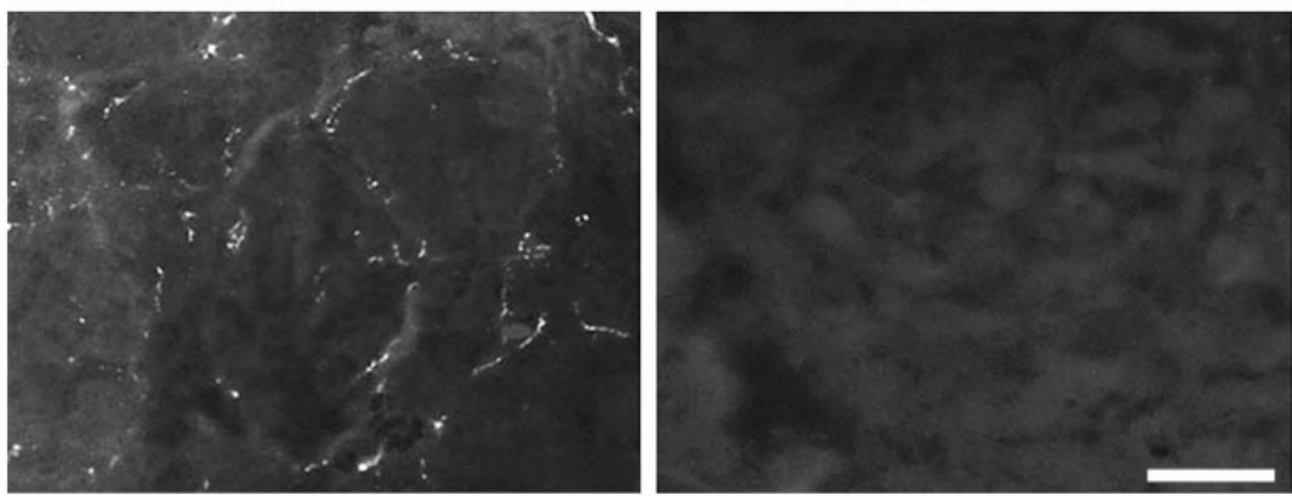

\section{TH -/-}

Figure 3. Catecholamine histofluorescence in the sweat gland innervation of albino $\mathrm{TH}$ wild-type and $\mathrm{TH}$ null mice at $\mathrm{P} 10$. Intense catecholamine histofluorescence is present in the developing sweat gland innervation of albino $\mathrm{TH}$ wild-type mice. In contrast, no catecholamine histofluorescence can be detected in the sweat gland innervation of albino $\mathrm{TH}$ null mice. Scale bar, $20 \mu \mathrm{m}$.

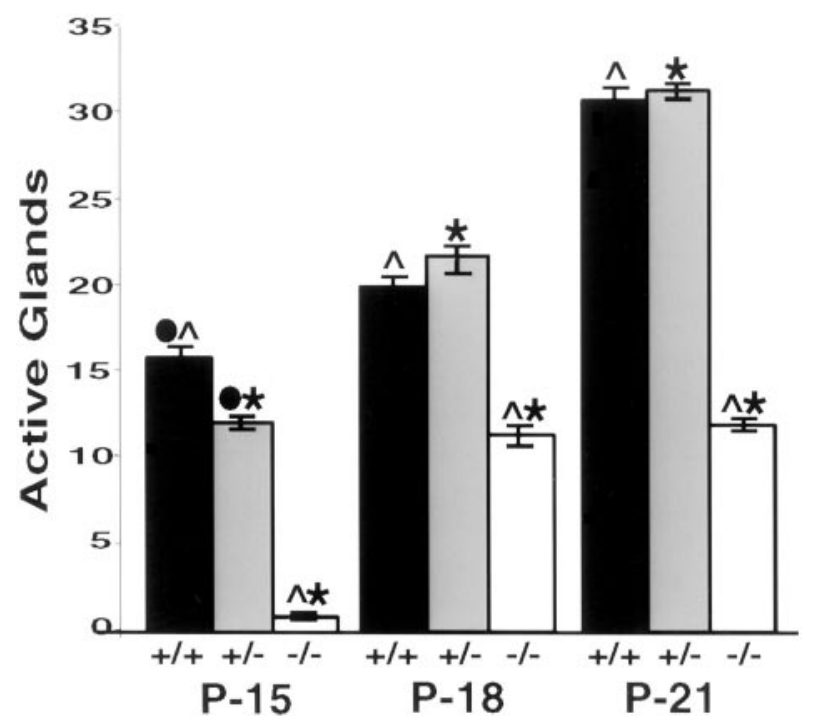

Figure 4. Quantitation of secretory responsiveness in pigmented TH null mice. The number of active glands was determined by counting the pores formed in the two interdigital footpads of pigmented mice that were wild-type at the TH locus $(+/+)$, heterozygous for the $\mathrm{TH}$ mutation $(+/-)$, or TH null $(-/-)$ at the ages indicated. Ât P15 and P18, fewer glands were active in the $\mathrm{TH}+/-$ mice than in wild-type mice. At all ages, only one or two active glands were detected in the TH null mice. Error bars represent SEM. ANOVA and post hoc pairwise comparisons with Sheffe's $F$ test ( $\alpha=$ 0.05 for all tests) indicated significant differences among all three genotypes at P15 and between the TH null and TH wild-type, heterozygote mice at both P18 and P21. Significant differences are indicated as 0 , between $+/+$ and $+/-$; $\wedge$, between $+/+$ and $-/-$; $*$, between $+/-$ and $-/-$.

TH wild-type mice (see Figs. 6, 9). To ascertain whether both myoepithelial and secretory cells develop in the absence of catecholamines, we examined sections of sweat glands from P21 albino TH wild-type and TH null mice for the expression of differentiated properties that characterize these cell types. Smooth muscle actin is expressed in myoepithelial cells. $\mathrm{Na}^{+}-\mathrm{K}^{+}$ATPase, which is required for secretory activity (Cook et al., 1994), and aquaporin 5, a water channel that allows rapid osmotic water flow in epithelial cells (Deen and van Os, 1998), are expressed in secretory cells. The relative proportions of the two cell types and the amount of histochemical activity or intensity of immunoreactivity of the labeled cells were similar in albino TH wild-type and null mice (Fig. 8).

\section{Catecholamines are not required for the appearance of cholinergic and peptidergic properties in sweat gland innervation}

In culture sweat glands require adrenergic receptor activation to produce cholinergic differentiation factor, which in turn induces cholinergic properties in the sympathetic neurons innervating them (Habecker et al., 1996, 1997). It is possible that the absence of a secretory response in albino $\mathrm{TH}$ null mice was a consequence of the absence of cholinergic function in the gland innervation, which is essential for secretory responsiveness (Grant et al., 1995). Therefore, we examined the developing sweat gland innervation for the appearance of two cholinergic markers, VAChT and acetylcholinesterase activity. VAChT is an excellent indicator of cholinergic function because the coding sequence for VAChT, which transports acetylcholine into synaptic vesicles, resides within the first intron of the ChAT gene (Bejanin et al., 1994; Erickson et al., 1994), and expression of the two genes is coordinately regulated in sympathetic neurons (Berrard et al., 1993; Misawa et al., 1995). In addition to cholinergic markers, the mature sweat gland innervation contains immunoreactivity for two neuropeptides, VIP and CGRP. VIP is induced in sympathetic neurons by cholinergic differentiation factors in vitro (Nawa et al., 1991; Rao and Landis, 1993) and by sweat glands in vivo (Schotzinger and Landis, 1990; Schotzinger et al., 1994).

When we compared the neurotransmitter properties of the sweat gland innervation of albino $\mathrm{TH}$ null mice with those of albino $\mathrm{TH}$ wild-type mice, we found that they were indistinguishable (Fig. 9). Robust VAChT immunoreactivity was evident at P21 in both genotypes. It was possible, however, that the time at which VAChT became detectable differed. At P7, the innervation of the TH null and wild-type mice had comparable levels of staining. Furthermore, there was no detectable difference in the expression of acetylcholinesterase activity, VIP, or CGRP in the sweat gland innervation of albino wild-type and $\mathrm{TH}$ null mice at P21. These results suggest that gland-dependent changes in the transmitter properties of the sweat gland innervation occur normally in the absence of catecholamines and that the glands require adrenergic as well as cholinergic activation to become competent to sweat.

\section{DISCUSSION}

Our findings demonstrate that the acquisition of secretory responsiveness by mouse sweat glands depends on the presence of catecholamines in the developing sympathetic innervation. That catecholamines are necessary is illustrated by examination of a transgenic mouse line in which the catecholamine content of sympathetic innervation is virtually eliminated because both $\mathrm{TH}$ and tyrosinase, which can serve as an alternative source of DOPA for catecholamine synthesis, are absent (Rios et al., 1999). Albino TH null mice, which have $<0.3 \%$ of the normal catecholamine content in hairy skin and no detectable catecholamine histofluorescence in footpad skin, possess on average fewer than one active gland in the two interdigital hind footpads at P21, when many glands are active in control mice. Acquisition of the secretory response can be supported by relatively small amounts of catecholamines. We charted the development of secretory responsiveness in $\mathrm{TH}$ null mice in which catecholamine levels were increased by two means: treatment of albino TH null mice with exogenous DOPA during the second and third postnatal weeks (Zhou and Palmiter, 1995) and expression of the $\mathrm{TH}$ null mutation in pigmented (tyrosinase- 


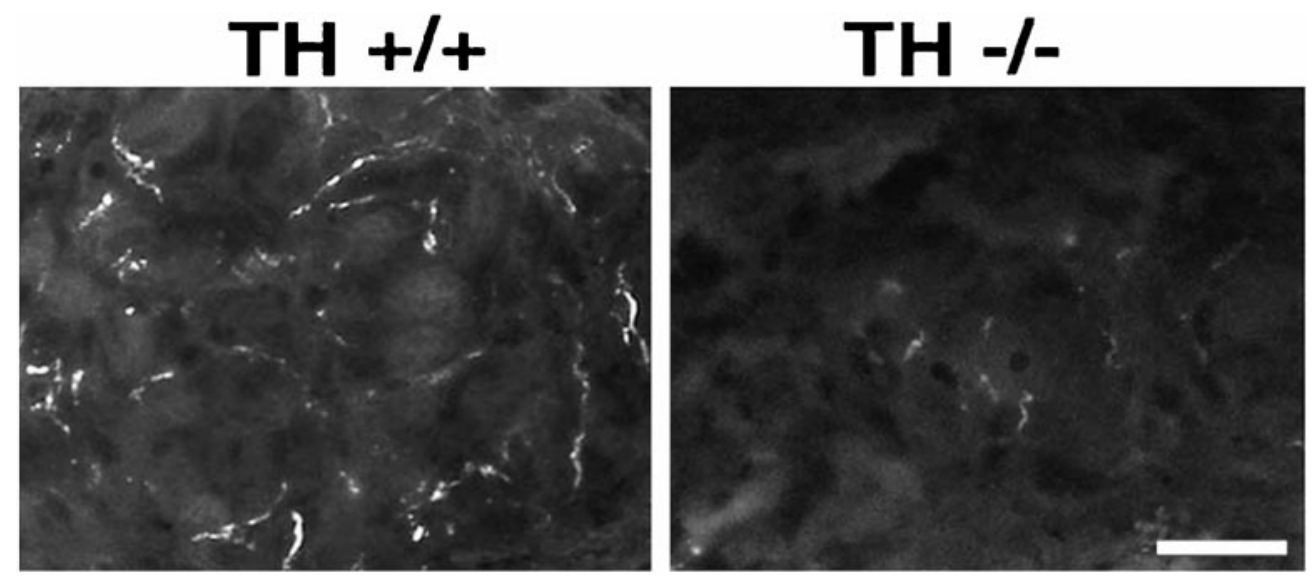

Figure 5. Catecholamine histofluorescence in the sweat gland innervation of pigmented $\mathrm{TH}$ wild-type and $\mathrm{TH}$ null mice. Intense catecholamine histofluorescence is present in the sweat gland innervation of $\mathrm{P} 10$ pigmented $\mathrm{TH}$ wild-type mice. Faint catecholamine histofluorescence can be detected in the sweat gland innervation of pigmented TH null mice at the same age. Scale bar, $20 \mu \mathrm{m}$.
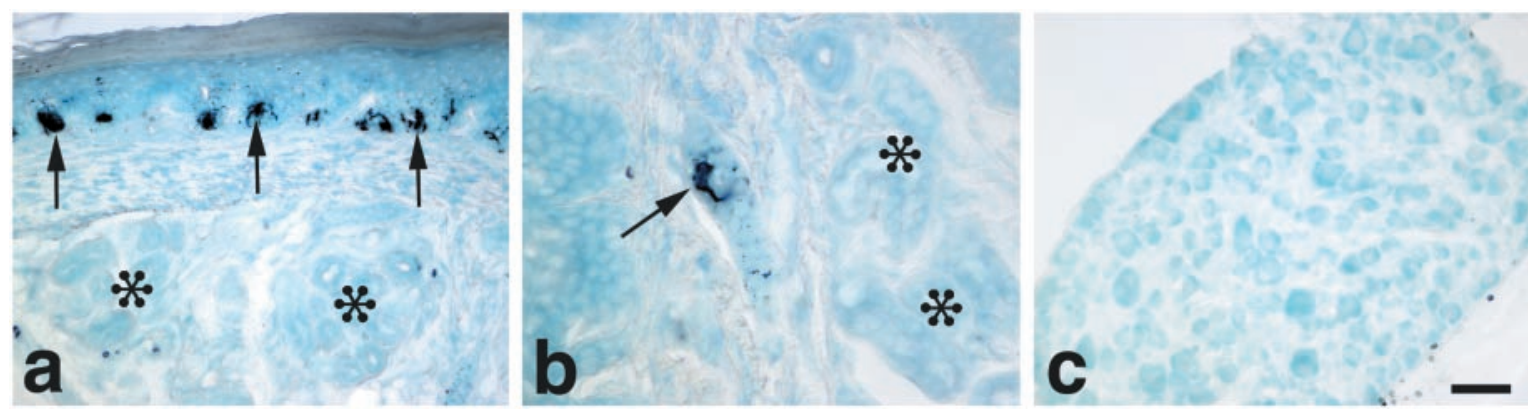

Figure 6. Presence of melanocytes in the footpads of P10 pigmented TH null mice. Melanocytes were identified using a histochemical reaction for tyrosinase. $a$, In footpads of pigmented mice, numerous darkly stained melanocytes (arrows) were present at the intersection of the epidermis and dermis, adjacent to sweat glands (asterisks). $b$, At higher magnification, the melanocyte cell body and processes (arrow) can be distinguished from melanin granule in cells of the secretory duct, which extends from the dermis on the left down toward the sweat gland (asterisks). No reaction product can be detected in the sympathetic innervation of the sweat glands. $c$, Neurons in lower lumbar sympathetic ganglia, which contain neurons that innervate the footpad sweat glands, were devoid of tyrosinase staining. Scale bar: $a, c, 10 \mu \mathrm{m} ; b, 20 \mu \mathrm{m}$.

containing) mice (Rios et al., 1999). In both cases, significantly more glands are active compared with untreated albino TH null mice. Thus, not only are catecholamines required for secretory responsiveness, but the time of onset and the extent of secretory responsiveness depend on catecholamine levels. Developing and mature sweat gland cells possess at least two adrenergic receptors, $\alpha_{1}$ and $\beta_{2}$ (Habecker et al., 1996). Each receptor activates the predicted second messenger system: $\alpha_{1}$ receptors stimulate phospholipase $C$, and $\beta_{2}$ receptors stimulate adenylyl cyclase activity. Induction of secretory responsiveness could require the activation of either one or both of these receptors.

Findings from previous cell culture studies suggested that catecholamines would influence secretory responsiveness indirectly by inducing the production of cholinergic differentiation factor by sweat glands (Habecker and Landis, 1994). Cholinergic innervation is required for the acquisition of secretory responsiveness (Stevens and Landis, 1987; Grant et al., 1995). The developing sweat gland innervation, however, is catecholaminergic (Landis and Keefe, 1983; Leblanc and Landis, 1986). Sweat glands induce cholinergic function in the sympathetic neurons that innervate them through production of a cholinergic differentiation factor (Schotzinger and Landis, 1988; Habecker et al., 1995; Guidry and Landis, 1998). In culture, catecholaminergic innervation is required to induce gland cells to produce cholinergic differentiation factor (Habecker and Landis, 1994; Habecker et al., 1995). Taken together, these findings predicted that sweat glands in albino $\mathrm{TH}$ null mice, which lacked catecholaminergic innervation, would not produce cholinergic differentiation factor, and therefore their innervation would not acquire the cholinergic function necessary to trigger the acquisition of secretory responsiveness.

When we examined the transmitter-related properties of the sweat gland innervation of albino TH null mice, however, we found that they were indistinguishable from those of albino TH wild-type mice. Immunoreactivity for VAChT appeared at the same time in albino TH null mice as in wild-type mice. Furthermore, other markers indicative of the target-dependent change in neurotransmitter phenotype, including acetylcholinesterase, VIP, and CGRP, were evident. These findings provide evidence that catecholamines are not responsible for triggering and/or maintaining production of the sweat gland-derived cholinergic differentiation factor in vivo. The signal(s) that regulate production of the cholinergic factor by sweat glands remains a puzzle. Elimination of sympathetic innervation in neonatal rats significantly decreases the cholinergic inducing activity that can be extracted from adult footpads (Habecker and Landis, 1994), suggesting that production of the cholinergic differentiation factor by sweat glands in vivo depends on innervation. Consistent with this notion, sweat gland cells cease factor production after dissociation and growth in the absence of sympathetic neurons. One possible explanation for the difference between the earlier in vitro findings and the new results obtained in vivo is that a neural-derived signal distinct from catecholamines induces factor production in vivo. Alternatively, the activity extracted from footpads may not accurately reflect production of the cholinergic differentiation factor by sweat glands; therefore, factor production may not be innervation-dependent in vivo.

Many, but not all, aspects of sweat gland development occur normally in the absence of catecholaminergic innervation. Numerous secretory coils are present in the footpads of albino $\mathrm{TH}$ null mice, and cells in the coils express markers of differentiated myoepithelial or secretory cells. The finding that morphological development of sweat glands can proceed without catecholaminergic innervation is consistent with previous analyses of sweat gland development in rat pups whose sympathetic nervous systems were destroyed at birth by treatment with the adrenergic neurotoxin 6-hydroxydopamine (6-OHDA), eliminating both initial catecholaminergic and subsequent cholinergic innervation (Yodlowski 


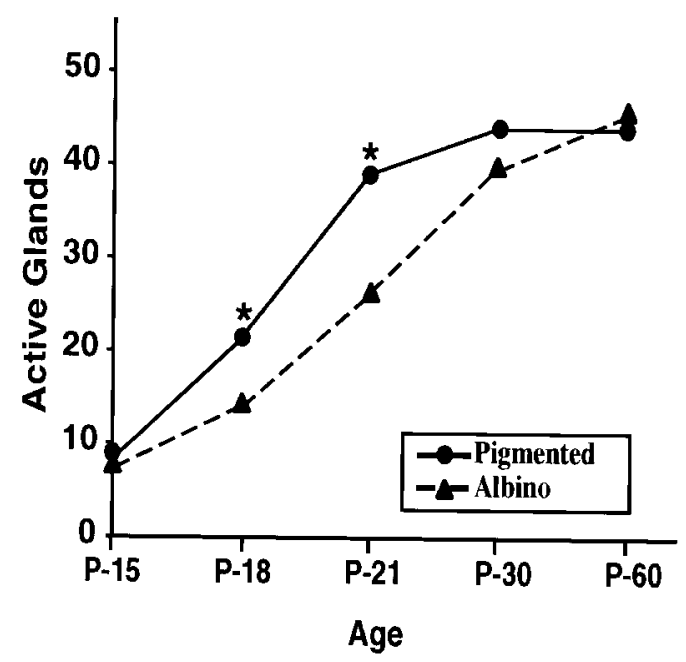

Figure 7. Quantitation of secretory responsiveness in congenic albino and pigmented C57BL/6J mice. The number of active glands was determined by counting the pores formed in the two interdigital hind footpads of albino and pigmented C57BL/6J mice at the ages indicated. At P15, secretory responsiveness was indistinguishable in the albino and pigmented mice. At P18 and P21, however, significantly fewer glands were active in the albino mice than in the pigmented mice. *Differences were considered statistically significant by Student's $t$ test $(\alpha=0.05)$.

et al., 1984). Not only does gland morphogenesis occur normally in 6-OHDA-treated rat pups, but so does the development of adrenergic and muscarinic receptors and their coupling to second messenger systems (Grant and Landis, 1991; Grant et al., 1991; Habecker et al., 1996). Despite the fact that glands appear to develop normally in albino TH null mice and 6-OHDA-treated rats, in neither case are the sweat glands competent to produce sweat droplets in response to treatment with a muscarinic agonist; i.e., they fail to mature functionally.

Our data provide evidence that catecholamines, like acetylcholine, are necessary to trigger the final differentiation of sweat glands required for secretory responsiveness. We do not know what aspect(s) of secretory responsiveness is regulated by catecholamines and acetylcholine. On the basis of studies of sweat secretion (Landis, 1999) and extrapolating from what is known of the regulation of fluid secretion in salivary glands (Baum, 1993; Cook et al., 1994), several steps are likely in the coupling of muscarinic receptor stimulation to sweat secretion. Rat sweat glands express predominantly the M3 receptor subtype (Grant et al., 1991). Receptor activation stimulates phospholipase $C$ and increases phosphoinositide turnover (Grant et al., 1991). In salivary glands and presumably sweat glands, calcium is then released from internal stores and also enters from the extracellular space. Increased cytoplasmic calcium concentration activates several ion channels, including $\mathrm{K}^{+}$and $\mathrm{Cl}^{-}$channels and the $\mathrm{Na}^{+}-\mathrm{K}^{+}-2 \mathrm{Cl}^{-}$ cotransporter and possibly translocation of the aquaporin 5 water channel to the plasma membrane (Ishikawa et al., 1998). Although we have not examined individual steps in the stimulus-secretion coupling in albino TH null mice, we know from previous studies that muscarinic receptor expression, coupling to phospholipase C, and stimulation of phosphoinositide metabolism are normal in sweat glands that have received neither adrenergic nor cholinergic innervation (Yodlowski et al., 1984; Grant and Landis, 1991). Taken together, our studies are consistent with the notion that the acquisition of one or more steps in stimulus-secretion coupling downstream from second messenger generation requires acetylcholine and catecholamines. Whether both transmitters control the same or different steps remains to be determined.

The present studies also revealed that DOPA synthesized by tyrosinase can contribute to catecholaminergic function. Although the secretory response is barely detectable in tyrosinase-deficient albino $\mathrm{TH}$ null mice at $\mathrm{P} 21$, pigmented $\mathrm{TH}$ null mice have many active glands. Therefore, catecholamines produced via tyrosinase support the development of significant secretory responsiveness when TH is absent. Surprisingly, DOPA produced by tyrosinase can also contribute to catecholaminergic function when $\mathrm{TH}$ is present. Although secretory responsiveness of pigmented mice heterozygous for the null $\mathrm{TH}$ allele $(\mathrm{TH}+/-)$ was identical to that of wild-type mice $(\mathrm{TH}+/+)$, fewer glands were active in albino

\section{Smooth Muscle $\mathrm{Na}^{+}-\mathrm{K}^{+}$ATPase} Actin
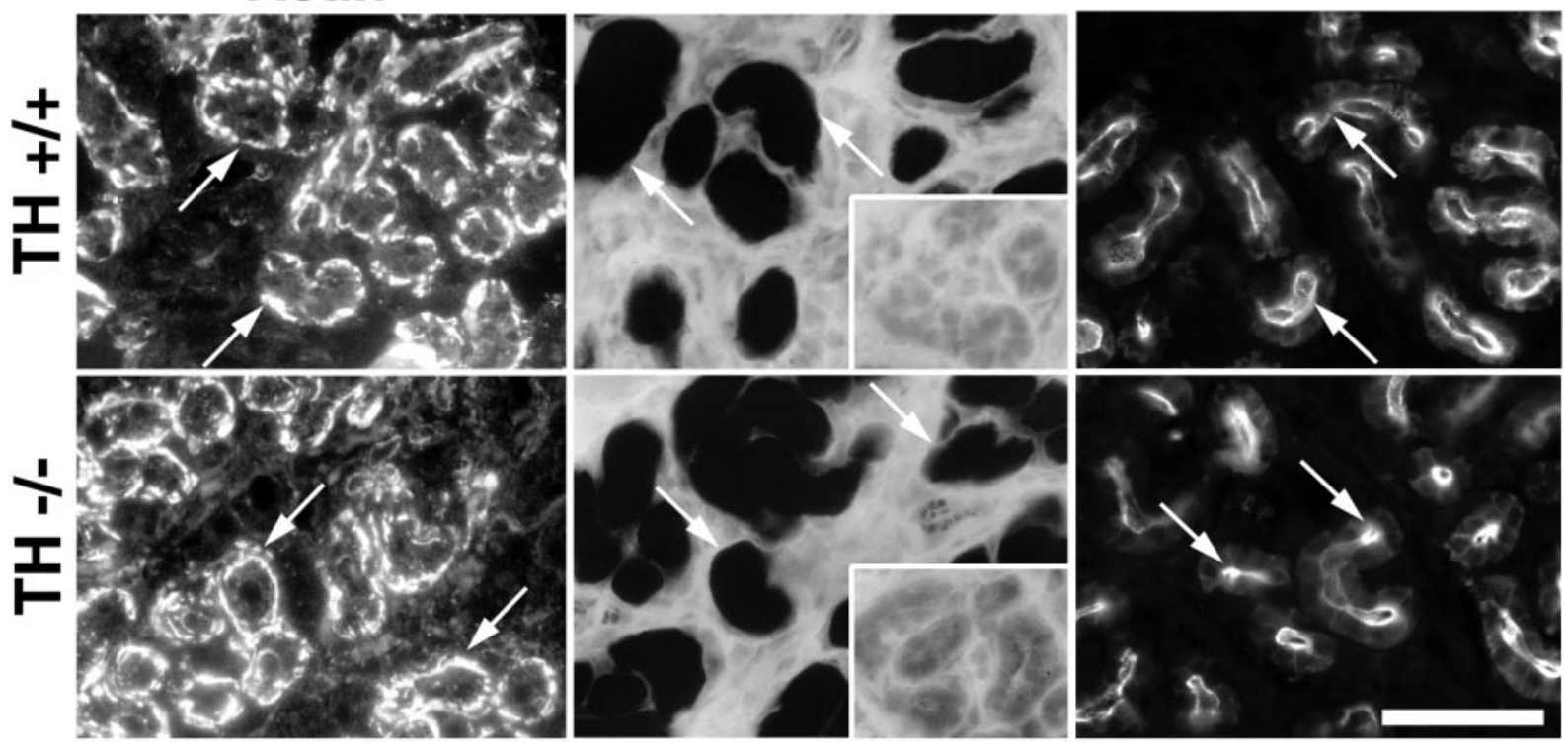

Figure 8. Histochemical and immunocytochemical characterization of cells present in the secretory tubules in albino TH wild-type and TH null mice at P21. Myoepithelial cells (arrows) were identified by their immunoreactivity for smooth muscle actin. Secretory cells (arrows) were identified using a polyclonal antiserum that recognizes aquaporin 5 and a histochemical assay for the $\mathrm{Na}^{+}-\mathrm{K}^{+}$ATPase. The specificity of the ATPase reaction is determined by its sensitivity to ouabain. No differences were evident in the expression of the three markers when wild-type and TH null sweat glands were compared. Scale bar, $20 \mu \mathrm{m}$. 
$\mathrm{TH}+/+$
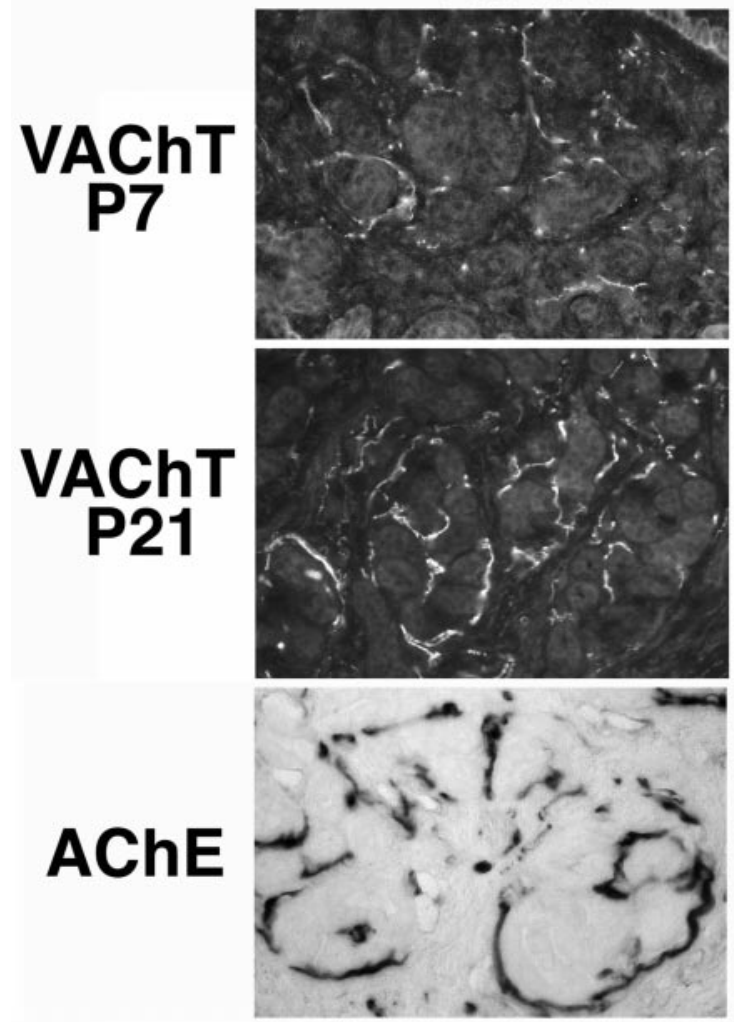

VAChT P7

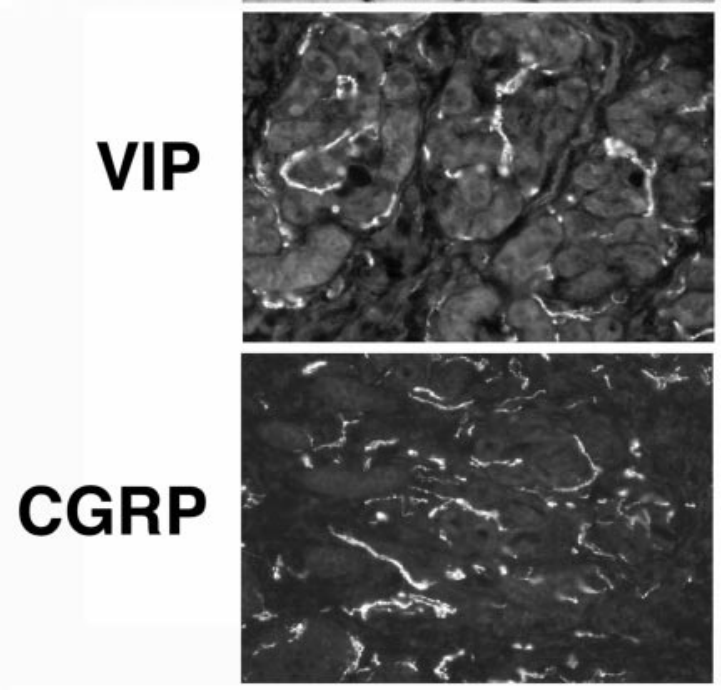

TH -/-
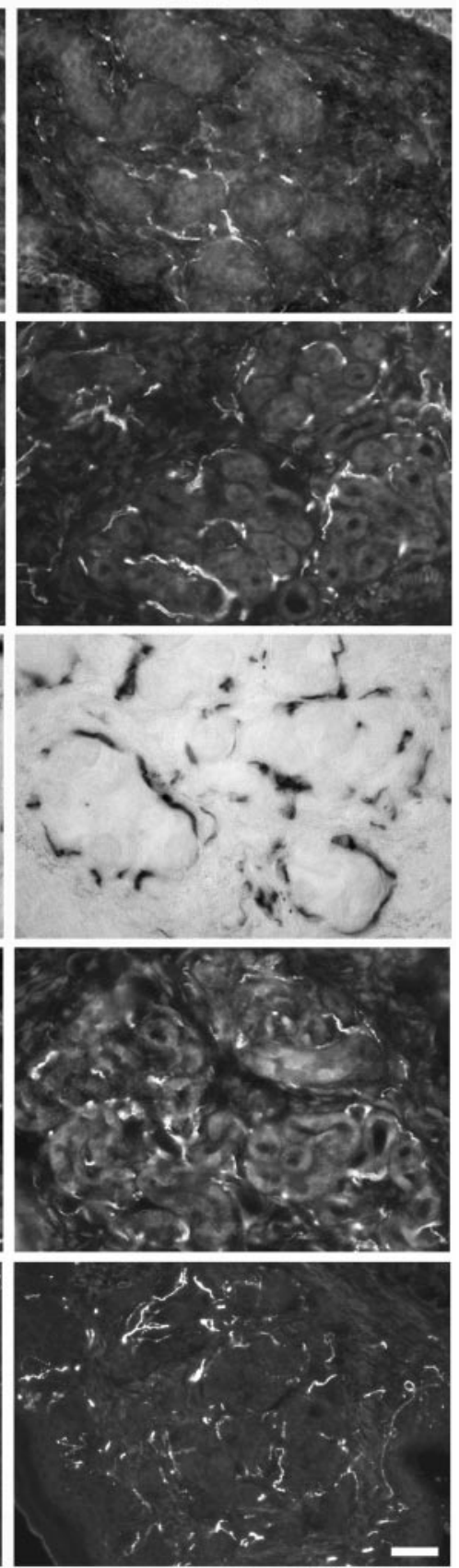

Figure 9. Development of neurotransmitterrelated properties in the sweat gland innervation of albino TH null mice. The development of VAChT immunoreactivity was compared between albino TH wild-type and TH null mice at P7 and P21. No differences were evident in either the extent of the sympathetic plexus or the intensity of immunoreactivity when wild-type and TH null mice were compared at each age. In addition, acetylcholinesterase activity and immunoreactivity for VIP and CGRP were expressed at normal levels in albino TH null mice. In addition, the secretory coils in the albino $\mathrm{TH}$ null mice were similar in appearance to those of the albino TH wild-type. Scale bar, $10 \mu \mathrm{m}$. heterozygotes $(\mathrm{TH}+/-)$ than in wild-type mice $(\mathrm{TH}+/+)$ at $\mathrm{P} 15$ and P18. This difference suggests that tyrosinase can compensate for one inactive $\mathrm{TH}$ allele. Finally, a transient but significant difference was observed in the development of the secretory response in pigmented and albino congenic C57BL/6J mice. Although differences in function attributable to catecholamines derived from DOPA produced by tyrosinase can be detected in our experimental paradigm, it is unclear that tyrosinase-derived DOPA plays a physiologically significant role in this system or in others.

The likeliest source of the tyrosinase-derived DOPA is melanocytes. Tyrosinase is expressed at very high levels in melanocytes in the skin. In the footpad, they are present at the epidermal-dermal border adjacent to the sweat glands. Tyrosinase catalyzes the hydroxylation of tyrosine to form DOPA, which is freely diff usible (Jackson et al., 1994; Spritz and Hearing, 1994; del Marmol and Beermann, 1996). DOPA levels are 100-fold higher in the hairy skin of pigmented pups than in albino pups at P15 and 10-fold higher in the plasma of pigmented wild-type animals at P7 and p15 than in albino animals (G. Eisenhofer, personal communication), suggesting that tyrosinase in melanocytes is an important source of DOPA in skin and in plasma. Thus, during development, DOPA derived from tyrosinase could diffuse from melanocytes in footpad skin or from plasma to sympathetic nerve terminals containing $\mathrm{D} \beta \mathrm{H}$, which would result in the synthesis of dopamine and norepinephrine. Although tyrosinase transcripts and protein have been detected in neonatal and adult mouse brain, they are present at much lower levels than in skin (Tief et al., 1996a,b). Tyrosinase activity has not been detected in mouse brain, and we did not observe tyrosinase in sweat gland innervation or sympathetic neurons. Although we cannot exclude the possibility that sympathetic neurons contain low levels of tyrosinase, there is no evidence to support this notion.

The sympathetic innervation of sweat glands is unusual because it is initially catecholaminergic but becomes cholinergic after in- 
teractions with the target tissue. It has been known for some time that acetylcholine is essential for synaptic function, because activation of muscarinic receptors is required for sweat secretion (Hayashi and Nakagawa, 1963; Kennedy and Sakuta, 1984; Stevens and Landis, 1987). More recently, it has become clear that cholinergic innervation is also required for the acquisition and maintenance of secretory responsiveness (Grant et al., 1995). Whether catecholamines also played a role in the development and/or function of this synapse has been less clear (Habecker and Landis, 1994; Weihe et al., 1996; Tsahai Tafari et al., 1997). The present studies provide evidence that although acetylcholine is necessary, it is not sufficient. Although catecholamines cannot elicit sweat secretion, they, like acetylcholine, are necessary to trigger the final maturation of the glands and to induce secretory responsiveness. Thus, both transmitter phenotypes play a crucial role in the development of sweat glands and establishment of this synapse.

\section{REFERENCES}

Baum B (1993) Principles of salivary secretion. Ann NY Acad Sci 694:17-23.

Bejanin S, Cervini R, Mallet J, Berrard S (1994) A unique gene organization for two cholinergic markers, choline acetyltransferase and a putative vesicular transporter of acetylcholine. J Biol Chem 269:21944-21947.

Berrard S, Faucon Biguet N, Houhou L, Lamouroux A, Mallet J (1993) Retinoic acid induces cholinergic differentiation of cultured newborn rat sympathetic neurons. J Neurosci Res 35:382-389.

Cook DI, Van Lennep EW, Roberts ML, Young JA (1994) Secretion by the major salivary glands. In: Physiology of the gastrointestinal tract, Ed 3 (Johnson L, ed), pp 1061-1117. New York: Raven.

Deen PM, van Os CH (1998) Epithelial aquaporins. Curr Opin Cell Biol $10: 435-442$

de La Torre JC (1980) An improved approach to histofluorescence using the SPG method for tissue monoamines. J Neurosci Methods 3:1-5.

del Marmol V, Beermann F (1996) Tyrosinase and related proteins in mammalian pigmentation. FEBS Lett 381:165-168.

Erickson JD, Varoqui H, Schafer MK-H, Modi W, Diebler M-F, Weihe E, Rand J, Eiden LE, Bonner TI, Usdin TB (1994) Functional identification of a vesicular acetylcholine transporter and its expression from a "cholinergic" gene locus. J Biol Chem 269:21929-21932.

Grant MP, Landis SC (1991) Developmental expression of muscarinic cholinergic receptors and coupling to phospholipase $\mathrm{C}$ on rat sweat glands are independent of innervation. J Neurosci 11:3772-3782.

Grant MP, Landis SC, Siegel RE (1991) The molecular and pharmacological properties of muscarinic cholinergic receptors expressed by rat sweat glands are unaltered by denervation. J Neurosci 11:3763-3771.

Grant MP, Francis NJ, Landis S (1995) The role of acetylcholine in regulating secretory responsiveness in rat sweat glands. Mol Cell Neurosci $6: 32-42$.

Guidry G, Landis SC (1995) Sympathetic axons pathfind successfully in the absence of target. J Neurosci 15:7565-7574.

Guidry G, Landis SC (1998) Target-dependent development of the vesicular acetylcholine transporter in rodent sweat gland innervation. Dev Biol 199:175-184.

Gurr E (1958) Methods of analytical histology and histochemistry, p 205. London: Leonard Hill.

Guth L, Albers RW (1974) Histochemical demonstration of $(\mathrm{Na}+\mathrm{-K}+)-$ activated adenosine triphosphatase. J Histochem Cytochem 22:320-326.

Habecker BA, Landis SC (1994) Noradrenergic regulation of cholinergic differentiation. Science 264:1602-1604.

Habecker BA, Tresser SJ, Rao MS, Landis SC (1995) Production of sweat gland cholinergic differentiation factor depends on innervation. Dev Biol 167:307-316.

Habecker BA, Malec N, Landis SC (1996) Differential regulation of adrenergic receptor development by sympathetic innervation. J Neurosci $16: 229-237$.

Habecker BA, Symes AJ, Stahl N, Francis NJ, Economides A, Fink J, Yancopoulos GD, Landis S (1997) A sweat gland-derived differentiation activity acts through known cytokine signaling pathways. J Biol Chem 272:30421-30428.

Hayashi H, Nakagawa T (1963) Functional activity of the sweat glands of the albino rat. J Invest Dermatol 41:365-367.

He X, Tse C, Donowitz M, Alper S, Gabriel S, Baum B (1997) Polarized distribution of key membrane transport proteins in the rat submandibular gland. Eur J Physiol 433:260-268.

Ishikawa Y, Eguchi T, Skowronski MT, Ishida H (1998) Acetylcholine acts of M3 muscarinic receptors and induces the translocation of aquaporin5 water channel via cytosolic $\mathrm{Ca}^{2+}$ elevation in rat parotid glands. Biochem Biophys Res Commun 245:835-840.

Jackson IJ, Budd P, Horn J, Johnson R, Raymond S, Steel K (1994) Genetics and molecular biology of mouse pigmentation. Pigm Cell Res 7:73-80.
Kennedy WR, Sakuta M (1984) Collateral reinnervation of sweat glands. Ann Neurol 15:73-78.

Kennedy WR, Sakuta M, Quick DC (1984) Rodent eccrine sweat glands: a case of multiple efferent innervation. Neuroscience 11:741-749.

Kobayashi K, Morita S, Sawada H, Miziguchi T, Yamada K, Nagatsu I, Hata T, Watanabe Y, Fujita K, Nagatsu T (1995) Targeted disruption of the tyrosine hydroxylase locus results in severe catecholamine depletion and perinatal lethality in mice. J Biol Chem 270:27235-27243.

Landis SC (1999) Development of muscarinic receptors and regulation of secretory responsiveness in rodent sweat glands. Life Sci 64:381-385.

Landis SC, Keefe D (1983) Evidence for neurotransmitter plasticity in vivo: developmental changes in the properties of cholinergic sympathetic neurons. Dev Biol 98:349-372.

Landis SC, Schwab M, Siegel RE (1988) Evidence for neurotransmitter plasticity in vivo: II. Immunocytochemical studies of rat sweat gland innervation during development. Dev Biol 126:129-138.

Langley JN (1922) The secretion of sweat. Part I. Supposed inhibitory nerve fibres on the posterior nerve roots. Secretion after denervation. J Physiol (Lond) 56:110-121.

Leblanc G, Landis SC (1986) Development of choline acetyltransferase activity in the cholinergic sympathetic innervation of sweat glands. J Neurosci 6:260-265.

Misawa H, Takahashi R, Deguchi T (1995) Coordinate expression of vesicular acetylcholine transporter and choline acetyltransferase in sympathetic superior cervical neurones. NeuroReport 6:965-968.

Nawa H, Yamamori T, Le T, Patterson PH (1991) The generation of neuronal diversity: analogies and homologies with hematopoiesis. Cold Spring Harb Symp Quant Biol 55:247-253.

Quick DC, Kennedy WR, Yoon KS (1984) Ultrastructure of the secretory epithelium, nerve fibers, and capillaries in the mouse sweat gland. Anat Rec 208:491-499.

Rao MS, Landis SC (1993) Cell interactions that determine sympathetic neuron transmitter phenotype and the neurokines that mediate them. J Neurobiol 24:215-232.

Rios M, Habecker B, Sasaoka T, Eisenhofer G, Tian H, Landis S, Chikaraishi D, Roffler-Tarlov S (1999) Catecholamine synthesis is mediated by tyrosinase in the absence of tyrosine hydroxylase. J Neurosci 19:3519-3526.

Roghani A, Shirzadi A, Butcher LL, Edwards RH (1998) Distribution of the vesicular transporter for acetylcholine in the rat central nervous system. Neuroscience 82:1195-1212.

Schotzinger R, Landis SC (1988) Cholinergic phenotype developed by noradrenergic sympathetic neurons after innervation of a novel cholinergic target in vivo. Nature 335:637-639.

Schotzinger R, Landis SC (1990) Acquisition of cholinergic and peptidergic properties by the sympathetic innervation of rat sweat glands requires interaction with normal target. Neuron 5:91-100.

Schotzinger R, Yin X, Landis SC (1994) Induction of a novel, targetappropriate neurotransmitter phenotype in sympathetic neurons following transplantation. J Neurobiol 25:620-639.

Spritz RA, Hearing VJ (1994) Genetic disorders of pigmentation. Adv Hum Genet 22:1-45.

Stevens LM, Landis SC (1987) Development and properties of the secretory response in rat sweat glands: relationship to the induction of cholinergic function in sweat gland innervation. Dev Biol 123:179-190.

Stevens LM, Landis SC (1988) Developmental interactions between sweat glands and the sympathetic neurons which innervate them: effects of delayed innervation on neurotransmitter plasticity and gland maturation. Dev Biol 130:703-720.

Tief K, Hahne M, Schmidt A, Beermann F (1996a) Tyrosinase, the key enzyme in melanin synthesis, is expressed in murine brain. Eur J Biochem 241:12-16.

Tief K, Schmidt A, Aguzzi A, Beermann F (1996b) Tyrosinase is a new marker for cell populations in the mouse neural tube. Dev Dyn 205:445-456.

Tsahai Tafari A, Thomas SA, Palmiter RD (1997) Norepinephrine facilitates the development of the murine sweat response but is not essential. J Neurosci 17:4275-4281.

Tyrrell S, Landis SC (1994) NPY and VIP expression in sympathetic neuroblasts and subsequent regulation of neuropeptide expression. J Neurosci 14:4529-4547.

Vilches J, Navarro X, Verdu E (1995) Functional sudomotor responses to cholinergic agonists and antagonists in the mouse. J Auton Nerv Syst 55:105-111.

Weihe E, Tao-Cheng JH, Schafer MK, Erickson JD, Eiden LE (1996) Visualization of the vesicular acetylcholine transporter in cholinergic nerve terminals and its targeting to a specific population of small synaptic vesicles. Proc Natl Acad Sci USA 93:3547-3552.

Yodlowski ML, Fredieu JR, Landis SC (1984) Neonatal 6-hydroxydopamine treatment eliminates cholinergic sympathetic innervation and induces sensory sprouting in rat sweat glands. J Neurosci 4:1535-1548.

Zhou Q, Quaife C, Palmiter R (1995) Targeted disruption of the tyrosine hydroxylase gene reveals that catecholamines are required for mouse fetal development. Nature 374:640-643.

Zhou Q-Y, Palmiter RD (1995) Dopamine-deficient mice are severely hypoactive, adipsic and aphagic. Cell 83:1197-1209. 